\title{
Electron-Energy Loss and Optical Spectroscopy of Hybrid Nanogap-Antennas on Different Substrates
}

\author{
T. Brintlinger ${ }^{1}$, A. A. Herzing ${ }^{2}$, J. P. Long ${ }^{3}$, R.M. Stroud ${ }^{1}$, I. Vurgaftman ${ }^{4}$ and B. S. Simpkins ${ }^{3}$ \\ ${ }^{1}$ Materials Division, U. S. Naval Research Laboratory, Washington, DC \\ 2. Materials Measurement Science Division, Material Measurement Laboratory, National Institute of \\ Standards and Technology, Gaithersburg, MD \\ ${ }^{3 .}$ Chemistry Division, U. S. Naval Research Laboratory, Washington, DC \\ 4. Optical Sciences Division, U. S. Naval Research Laboratory, Washington, DC
}

Hybrid systems which combine plasmonic metals and active dielectrics into single nanostructures have recently been designed theoretically [1] and realized experimentally [2]. Technologically, they hold great potential for optically modulated communication circuits and for photocatalysis to aid a broad range of energy production technologies $[3,4]$. Scientifically, the underlying physics of localized resonances in these systems can be probed with multiple theoretical and experimental approaches [5]. While the ultimate, diffraction-limited, resolution of conventional optical microscopy is not sufficient to map out the spatial distribution of these modes, characterization of these modes naturally lends itself to the high-spatial resolution and spectroscopic capabilities of a scanning transmission electron microscope (STEM) fitted with an electron energy loss spectrometer (EELS) [6, 7]. As such, we use coordinated optical spectroscopy, EELS, and numerical simulation to study nanogap hybrid antennas consisting of two metal nanostructures joined by a dielectric gap material.

Nanogap antennas, comprised of two Au nanorods with a uniform diameter of $\approx 65 \mathrm{~nm}$ (with individual rod lengths ranging from 105 to $130 \mathrm{~nm}$ ) joined by a CdS-filled $\approx 40 \mathrm{~nm}$ gap were fabricated using electrochemical deposition in anodized-aluminum-oxide (AAO) templates. Nanostructures were then released from the templates and cast onto a variety of substrates including electron-transparent nonporous silicon, silicon nitride, and lacey carbon substrates. The plasmonic resonances were then probed with EELS using a spherical aberration-corrected STEM with a monochromated source. With $<$ $2 \mathrm{~nm}$ spatial resolution and $\approx 150 \mathrm{meV}$ energy resolution, EELS results successfully revealed these highly localized excitations (Fig 1 and 2). Furthermore, we complement the EELS spectroscopy with dark-field optical scattering measurements and finite-difference time-domain (FTDT) simulations. All three approaches reveal three primary resonances which we will term longitudinal, gap, and transverse modes located at ca. 1.3, 1.9, and $2.3 \mathrm{eV}$, respectively. After background subtraction and principal component analysis, EELS results reveal spatial distributions for these modes consistent with FTDT results. Specifically, the longitudinal mode intensity is localized at the extreme ends of the antenna, the gap mode is spatially centered in the CdS-filled gap region between nanorods, and the transverse mode extends along the sides of the two rods. We will present detailed descriptions of fabrication and measurement techniques while discussing signal extraction and display [8].

\section{References:}

[1] A Alu \& N Engheta, Nat Photon 2 (2008), p. 307-310.

[2] BS Simpkins, et al., J Phys Chem C 117 (2013), p. 11843-11849.

[3] E Ozbay, Science 311 (2006), p. 189-193.

[4] Z Xuming, et al., Reports on Progress in Physics 76 (2013), p. 046401. 
[5] M Kauranen \& AV Zayats, Nat Photon 6 (2012), p. 737-748.

[6] M-W Chu, et al., Nano Letters 9 (2008), p. 399-404.

[7] BS Guiton, et al., Nano Letters 11 (2011), p. 3482-3488.

[8] Work at the Naval Research Laboratory was funded through the Office of Naval Research.

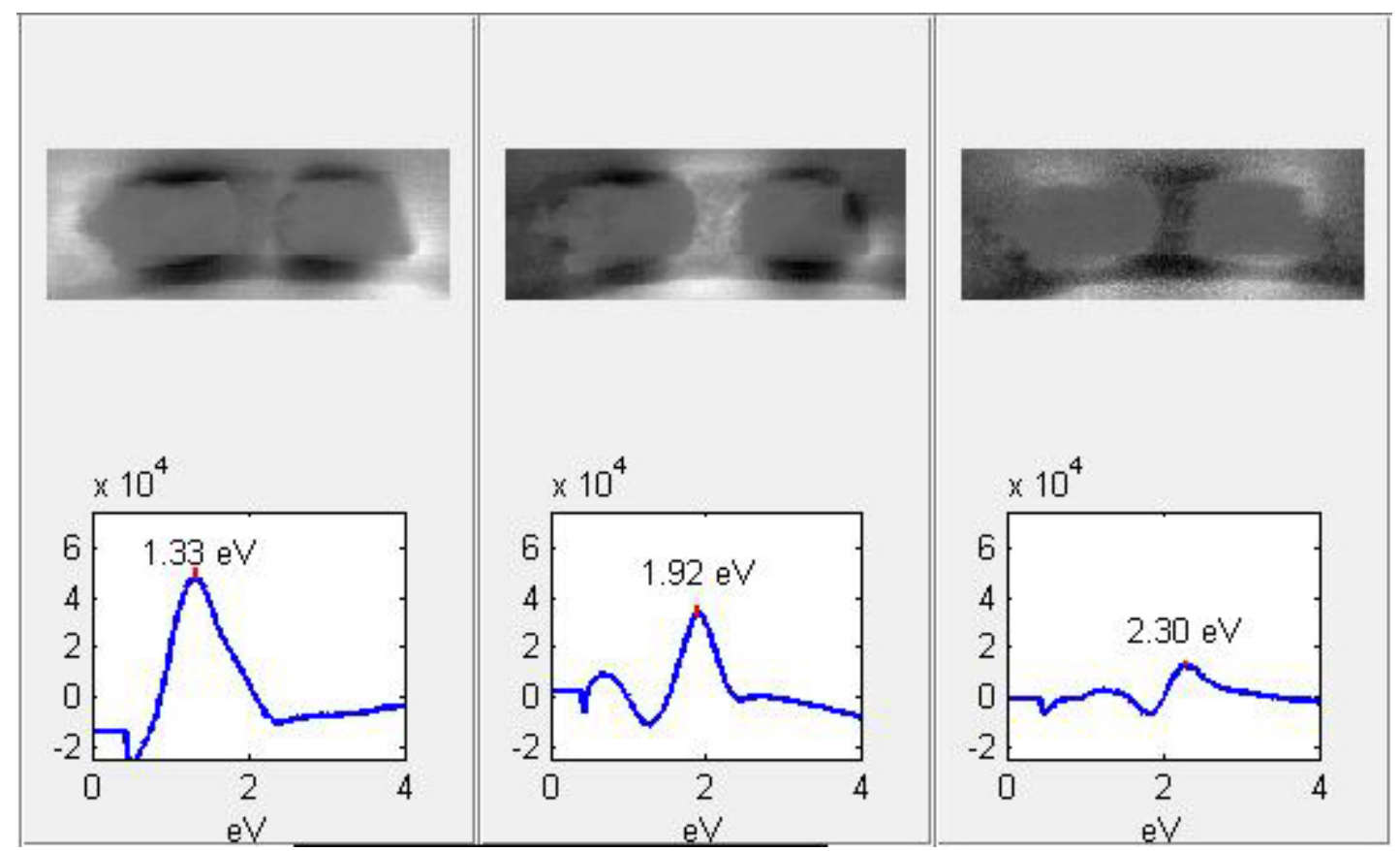

Figure 1. Nanogap antennas on lacey carbon: Nanogap antenna consist of long gold rods, with cadmium sulfide in 'gap' identified by principal component analysis and illustrated beneath each image.

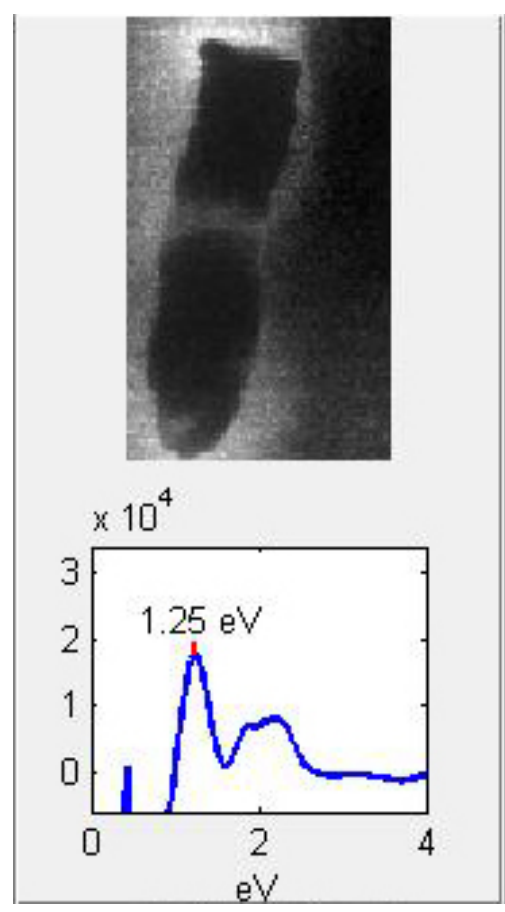

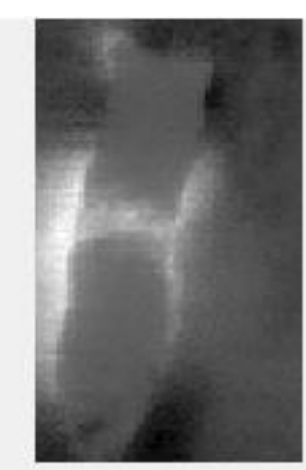

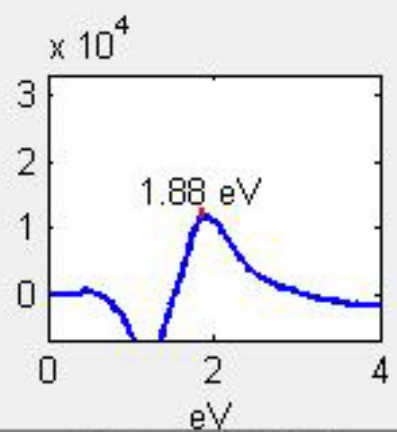

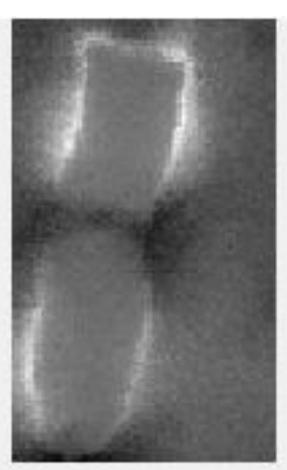

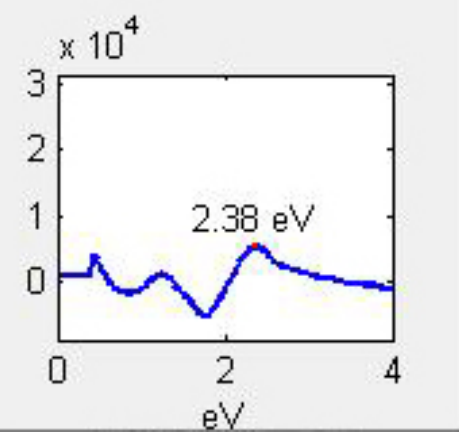

Figure 2. Nanogap antennas on silicon nitride, with same treatment as in Fig. 1. 